\title{
INCOME SMOOTHING, CUSTO DE CAPITAL DE TERCEIROS E ESTRUTURA DE CAPITAL NO BRASIL
}

\section{INCOME SMOOTHING, COST OF DEBT CAPITAL AND CAPITAL STRUCTURE IN BRAZIL}

MIGUEL ANGEL RIVERA CASTRO

Doutorando do Programa de Energia e Meio Ambiente da Universidade Federal da Bahia (UFBA). Professor substituto da Faculdade de Ciências Contábeis da Universidade Federal da Bahia. Praça da Piedade, 6, Centro - Salvador - BA - Brasil - CEP 40070-010 E-mail: marc@ufba.br

\section{ANTONIO LOPO MARTINEZ}

Doutor em Administração de Empresas pela Escola de Administração de Empresas de São Paulo da Fundação Getulio Vargas (FGV). Professor do Programa de Pós-Graduação da Fundação Instituto Capixaba de Pesquisa em Contabilidade, Economia e Finanças (Fucape). Av. Fernando Ferrari, 1358, Boa Vista - Vitória - ES - Brasil - CEP 29075-505 E-mail: lopo@fucape.br 


\section{RESUMO}

Neste artigo, usando uma amostra de companhias abertas brasileiras e os modelos propostos por Eckel (I98I) e Leuz, Nanda e Wysocki (2003) como proxies empíricas para mensurar income smoothing, os resultados dos testes indicam que as empresas que promovem income smoothing estão propensas a ter um menor custo de capital de terceiros e uma estrutura de capital com maior peso para endividamentos de longo prazo. Metodologicamente, utilizou-se um modelo de regressão de dados em painel tipo Nerlove para informações no período de 2003 a 2007. A análise defasada em um e dois períodos revela que o alisamento de resultados corrente provocará impacto no custo de capital futuro, bem como na definição da estrutura de financiamento da empresa. Os resultados, após a obtenção do controle da heterogeneidade e dos fatores conhecidos para explicar o custo de capital e a estrutura de capital, revelaram-se robustos, indicando a significância do fator income smoothing no que diz respeito a variáveis explicativas alternativas. A influência do fator suavização defasado em um e dois períodos possui amplitude comparável à influência de outros fatores, como tamanho e risco. Os resultados suportam a noção de que o fator income smoothing no Brasil representa um mecanismo information-signaling, tendo implicações no custo de capital e nas decisões de financiamento das empresas.

\section{PALAVRAS-CHAVE}

Income smoothing; Custo de capital de terceiros; Estrutura de capital; Gerenciamento de resultados; Lucro líquido.

\section{ABSTRACT}

In this paper using a sample of Brazilian companies and models proposed by Eckel (I98I) and Leuz, Nanda, and Wysocki (2003) as empirical proxies to measure income smoothing, test results indicate that companies that promote income 
smoothing are likely to have a lower cost of capital and a capital structure with greater percentage of long-term debt. The methodology used included a panel data Nerlove type for a period from 2003 to 2007 . The analysis lagged in one and two periods reveals that smoothing will impact on future cost of capital and the capital structure. Results after controlling heterogeneity and factors known to explain the cost of capital and capital structure proved to be robust, indicating the significance of income smoothing with respect to alternative explanatory variables. The influence of smoothing factor in one and two years delayed have comparable magnitude to other factors such as size and risk. The results support the notion that income smoothing in Brazil is an information-signaling mechanism, with implications for the cost of capital and the financing decisions.

\section{KEYWORDS}

Income smoothing; Cost of debt capital; Capital structure; Earnings management; Net income.

\section{INTRODUÇÃO}

O gerenciamento de resultados contábeis é uma questão que vem preocupando os pesquisadores há vários anos, existindo atualmente várias linhas de pesquisa nesse domínio. Nesse extenso tema, inscreve-se o denominado income smoothing. Em particular, o income smoothing constitui uma das vertentes do gerenciamento de resultados que tem atraído significativa atenção por parte da literatura de finanças e contabilidade dedicada ao earnings management. Com efeito, trata-se de uma tendência herdada do passado e plenamente vigente nos dias de hoje, que visa diminuir as possíveis flutuações do lucro para estabilizá-lo ao longo do tempo.

A maioria dos trabalhos publicados sobre o tema se centra em analisar os fatores que conduzem ao comportamento de smoothing ou contrastar a existência ou não desse fenômeno em diferentes setores. Na literatura internacional, os trabalhos que têm estudado a relação entre rentabilidades no mercado e o grau de income smoothing nas empresas são os de Michelson, Jordan-Wagner e Wootton (I995, I999), Booth, Kallunki e Martikainen (1996), Bin, Wan e Kamil (2000), Iñiguez e Poveda (2004), Bao e Bao (2004), Tan e Jamal (2006), Tucker e Zarowin (2006) e Grant, Parbonetti e Markarian (2007), que analisaram os mercados norte-americano, finlandês, malaio e espanhol. Se restringirmos a análise 
apenas aos aspectos relacionados ao longo prazo, a literatura internacional conta somente com os trabalhos de Michelson, Jordan-Wagner e Wootton (I995, I999) e Iñiguez e Poveda (2004) que chegam a conclusões díspares trabalhando com metodologias distintas.

Neste paper, realizou-se uma analise do efeito do income smoothing no mercado de ações brasileiro, com a intenção de verificar a associação entre o grau de income smoothing e seu efeito na estrutura de capital e no custo de capital de terceiros. Com a motivação e os objetivos expostos, o artigo estruturou-se em seções temáticas. Após a introdução, na segunda seção, apresentam-se a descrição do income smoothing e as métricas para sua detecção. A seguir, indicam-se o desenho da pesquisa e a metodologia de análise. Na quarta seção, são apresentados os resultados da análise empírica realizada. Por último, sintetizam-se as principais conclusões apreciadas no mercado acionário brasileiro.

\section{INCOME SMOOTHING}

\subsection{INCOME SMOOTHING E CUSTO DE CAPITAL DE TERCEIROS}

Collins e Kothari (I989), Easton e Zmijewski (I989) e Barth, Landsman e Wahlen (I995) demonstram que os preços das ações refletem um prêmio pelos riscos associados à variabilidade dos resultados e que os gestores podem reduzir os custos de capital usando seu poder discricionário na estimação de certos componentes de resultado para reduzir a sua variabilidade. A literatura denomina esse procedimento "alisamento de resultados", o qual é empregado nas seguintes situações: I. quando o resultado previamente apurado for alto, pela redução do resultado divulgado; e 2. quando o resultado previamente apurado for baixo, pelo aumento do resultado divulgado.

Os investidores e os credores percebem a dispersão dos resultados contábeis como medida de risco. Assim, as empresas que possuem menor variação nos seus resultados contábeis tendem a atrair mais investidores, bem como obter empréstimos a custos mais baixos. Essa percepção do mercado pode incentivar os gestores a tornar o resultado menos volátil, suavizando o seu fluxo de crescimento. Nesses casos, os gestores podem utilizar a modalidade de gerenciamento de resultados denominada alisamento de resultados.

A manipulação dos resultados por meio do alisamento de resultados, segundo Stolowy e Breton (2000), tem como objetivo a produção de fluxos de crescimento de lucros estáveis. Para que isso aconteça, é necessário que as empresas tenham lucros que permitam a criação de provisões para regular o fluxo quando necessário. 
Em uma pesquisa sobre o tema, Copeland (ig68) estabeleceu que o alisamento de resultados implica uma seleção repetitiva de mensuração contábil ou de uma regra de divulgação específica, cujo efeito é a divulgação de fluxos de lucros com variações menores do que seria mostrado de outra maneira.

Outros autores conceituaram essa modalidade de gerenciamento de resultados, entre os quais, pode-se citar Beidleman (I973) que definiu alisamento de resultados como a suavização intencional das flutuações no nível de resultado que geralmente é considerado normal para a empresa. A definição de Barnea, Ronen e Sadan (1976) é similar à anterior, diferenciando-se pela omissão do termo "intencional", que, na opinião dos autores, significaria conhecer a intenção da administração e ter a noção de que a intencionalidade não poderia ser testada por meio de estudo com dados empíricos não comportamentais.

Imhoff (198I) definiu alisamento de resultados como um caso especial de divulgação inadequada de informações financeiras e que essa técnica implica algum esforço deliberado para divulgar as informações financeiras, de maneira a reduzir artificialmente a variação dos fluxos de lucros. Para Beattie et al. (I994), a suavização pode ser vista em termos da redução na variação dos resultados em determinados períodos, ou dentro de um período, como o movimento em direção a um nível esperado de lucros.

Segundo Ronen e Yaari (2007), existem dois tipos de smoothing intencional: real e artificial. O real smoothing envolve a realização de decisões de produção e investimento que reduzem a variabilidade dos resultados contábeis. O artificial smoothing é obtido por meio de escolhas contábeis. Urge registrar que o smoothing pode ocorrer também de forma natural, sem nenhuma decisão oportunista, dada a reduzida variabilidade de resultados em determinados setores.

Independentemente da natureza do alisamento, a literatura acadêmica em modelagens teóricas sempre apontou que a redução da flutuação dos lucros (income smoothing) é benéfica para as firmas porque reduz o custo do capital de terceiros (TRUEMAN; TITMAN, I988). No mesmo sentido, Moyer e Shevlin (I995) argumentam que a pequena variabilidade dos resultados reduziria o risco sistemático da particular ação, em face da queda na covariância entre os retornos da firma e os retornos do mercado.

Diante do exposto, a questão de pesquisa é verificar no contexto brasileiro se o income smoothing é informativo para analistas financeiros e para o mercado de crédito, ou seja, se ele se reflete no custo de capital e no valor da empresa. Será que as empresas que apresentam resultados suavizados podem se beneficiar de um custo de capital de terceiros mais baixo? As empresas com reduzida variabilidade de resultados privilegiam o capital de terceiros em sua estrutura de capital? Em suma, esta investigação identifica o efeito do income smoothing sobre a estrutura de capital e o custo de capital de terceiros. Em definitivo, o efeito que se 
espera é que, diante do alisamento de resultados, ocorra uma menor percepção de risco por parte dos investidores, que se traduziria num menor spread e num aumento a propensão com o financiamento de recursos de terceiros.

\subsection{MÉTRICAS DE ALISAMENTO DE ECKEL (1981)}

A metodologia usada nos trabalhos sobre income smoothing se baseia no modelo dos coeficientes de variação proposto por Eckel (I98I) e pelos modelos de Leuz, Nanda e Wysocki (2003), e usado posteriormente por Booth, Kallunki e Martikainen (1996), Michelson, Jordan-Wagner e Wootton (I995, I999), Bin, Wan e Kamil (2000), Leuz, Nanda e Wysocki (2003) e Bao e Bao (2004).

Nesses trabalhos, é demonstrado que, se o lucro é função linear das vendas, o custo variável unitário se mantém constante no tempo, os custos fixos não diminuem e as vendas brutas não podem ser alisadas artificialmente; então, o coeficiente de variação das oscilações nas vendas é inferior ao coeficiente de variação das oscilações no lucro. Se isso não se cumpre, a variabilidade das oscilações no lucro é menor. Nesse caso, Eckel (I98I) demonstra que a empresa está alisando artificialmente lucro, ou seja:

$$
C V \Delta \% \text { lucro } \leq C V \Delta \% \text { vendas } \Rightarrow \text { Alisamento, }
$$

onde:

$\Delta \%$ lucro = Oscilação anual do lucro;

$\Delta \%$ vendas $=$ Oscilação anual das vendas.

$C V(\mathrm{x})=\sigma(\mathrm{x}) / \mu(\mathrm{x})$

Com base nesse raciocínio, relevantes trabalhos sobre alisamento têm sido publicados nos últimos vinte anos como os de Albrecht e Richardson (I990), Ashari et al. (I994), Booth, Kallunki e Martikainen (I996), Michelson, JordanWagner e Wootton (I995, I999), Bin, Wan e Kamil (2000) e Bao e Bao (2004), que calcularam uma medida de alisamento como um índice adimensional mediante o quociente dos coeficientes de variação, isto é:

$$
\mathrm{IA}_{\mathrm{I}}=\frac{C V \Delta \% \text { LucroLíquido }}{C V \Delta \% \text { Vendas }}
$$

Com base nessa medida, assumiu-se em todos esses trabalhos que um índice inferior a I, em valor absoluto, estaria indicando a presença de alisamento de resultados, já que o coeficiente de variação do lucro seria inferior ao das vendas, 
e Eckel (I98I) demonstrou que essa situação era provocada pelo alisamento por parte dos gestores. No entanto, nesta pesquisa foi modificado o referido modelo, excluindo as empresas, com um índice de alisamento (IA) entre o,90 e I,IO como área cinzenta. Esse procedimento é necessário para reduzir erros de classificação em harmonia com a metodologia de Chalayer (I994).

$$
0,9 \leq\left[\left|\frac{C V \Delta \% \text { LucroLíquido }}{C V \Delta \% \text { Vendas }}\right|\right] \leq \mathrm{I}, \mathrm{IO}
$$

\section{Alisamento $\leq \mid$ área cinza $\mid \leq$ Sem alisamento}

No presente trabalho, assume-se uma distinção estrita entre empresas alisadoras e não alisadoras segundo o índice de alisamento (IA), caso esteja acima ou abaixo da unidade, e assim é identificado um suporte empírico robusto para validar a hipótese de que a gestão se encontra motivada para reduzir a variabilidade dos resultados e dos fluxos de caixa como uma tentativa de reduzir o risco percebido da empresa.

A eleição desse procedimento de agrupação com base em um índice de alisamento está motivada fundamentalmente pelas seguintes razões:

- Para Bao e Bao (2004), o índice considera os efeitos agregados de todas as variáveis contábeis que tratam de alisar o lucro, descrevendo a pauta de comportamento de uma empresa em relação ao alisamento. As empresas não escolhem os procedimentos contábeis independentemente, elas consideram o efeito conjunto sobre o resultado que comunicam ao mercado. Por esse motivo, a eleição de uma única variável como instrumento de alisamento pode levar a conclusões erradas, já que seu efeito pode ver-se mitigado pelo efeito agregado de outras variáveis.

- Como apontam Albrecht e Richardson (I990), outra das vantagens dessa metodologia é que ela proporciona uma medida adimensional da variabilidade da amostra e permite comparações de variabilidades entre distintos grupos. Além disso, destaca-se sua utilidade na hora de comparar dados que têm distinta média e desvio padrão. Essas qualidades convertem o índice de alisamento num instrumento útil para a construção dos grupos em função do grau de alisamento. Entretanto, segundo Eckel (I98I), a principal deficiência da metodologia é não reconhecer como alisadoras as empresas que têm reduzido a variabilidade de seu lucro, mas não até o ponto de fazê-lo menos variável que as vendas. 
A metodologia dos coeficientes de variação relaciona o desvio padrão com a média de uma série de números representados pelos resultados publicados. Quanto mais esse coeficiente aproxima-se de zero, mais a série é alisada. Geralmente, o coeficiente de variação é calculado a partir das variações de resultado, o que supõe que se busca uma tendência crescente e alisada dos resultados (Eckel, I98I). No entanto, apesar do número importante de instrumentos disponíveis que permite aos gestores publicar uma série de resultados alisados, esses instrumentos não conseguem provavelmente assegurar um alisamento perfeito. Por conseguinte, o coeficiente de variação assim calculado nunca é nulo. Portanto, é preciso determinar a partir de qual limiar se observa a prática de alisamento de resultados. Esse limiar, se for preciso defini-lo, é necessariamente arbitrário. Como Eckel (I98I), podemos introduzir uma referência ao setor de atividade da firma: se o coeficiente de variação da firma for inferior ao coeficiente de variação médio do seu setor, a firma estará classificada no grupo das firmas que estão alisando intencionalmente seus resultados contábeis, mas esse limiar é ainda arbitrário.

\subsection{MÉTRICAS DE ALISAMENTO DE LEUZ, NANDA E WYSOCKI (2003)}

Essas métricas usadas para medir o alisamento correspondem à análise empírica feita por Leuz, Nanda e Wisocky (2003), Francis, Maydew e Sparks (2004), e Lopes, Tukamoto e Galdi (2007). Os executivos das empresas podem omitir mudanças do desempenho econômico de sua firma usando decisões operativas reais e escolhas sobre como evidenciar os relatórios financeiros (LEUZ; NANDA; WYSOCKI, 2003). Focada nas escolhas contábeis, a segunda métrica quer capturar o grau como os executivos praticam o alisamento, ou seja, como reduzem a variabilidade do lucro evidenciado pela alteração dos componentes contábeis do lucro, isto é, pelos ajustes do regime de competência. A métrica é computada pelo desvio padrão do lucro operacional dividido pelo desvio padrão do fluxo de caixa operacional. Essa variável, ao ser escalonada pelo fluxo de caixa operacional, controla de forma geral as diferenças de variabilidade do desempenho econômico. Valores pequenos dessa métrica indicam que, ceteris paribus, os executivos exercem discricionariedade ao fazerem o alisamento do lucro evidenciado nos relatórios contábeis.

O fluxo de caixa operacional é calculado indiretamente por meio da diminuição dos accruals ao lucro líquido. Em primeiro lugar, calculamos essa medida em cada firma de nossa amostra com uma estimação numa série temporal, isto é: 
FCO $=$ Lucro__ Líquido - Accruals,

onde:

Accruals $=\left\{\left[A C_{t}-\right.\right.$ Disp $\left.\left._{t}\right)-\left(P C_{t}-E m p C P_{t}\right)\right]-\left[\left(A C_{t-\mathrm{I}}-\operatorname{Disp}_{t-\mathrm{I}}\right)-\left(P C_{t-\mathrm{I}}\right.\right.$ $\left.\left.-E m p C P_{t-\mathrm{I}}\right)\right]-\operatorname{Depr} \&$ Amort $\left.t_{t}\right\}$

$\mathrm{AC}_{\mathrm{t}}=$ Ativo circulante no ano $\mathrm{t}$;

Disp $_{\mathrm{t}}=$ Disponibilidades no ano $\mathrm{t}$;

$\mathrm{PC}_{\mathrm{t}}=$ Passivo circulante no ano $\mathrm{t}$;

EmpCP $_{\mathrm{t}}=$ Empréstimos de curto prazo em $\mathrm{t}$;

$\mathrm{AC}_{\mathrm{t}-\mathrm{I}}=$ Ativo circulante no ano $\mathrm{t}-\mathrm{I}$;

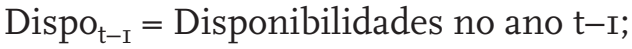

$\mathrm{PC}_{\mathrm{t}-\mathrm{I}}=$ Passivo circulante no ano $\mathrm{t}-\mathrm{I}$;

$\mathrm{EmpCP}_{\mathrm{t}-\mathrm{I}}=$ Empréstimos de curto prazo em $\mathrm{t}-\mathrm{I}$.

Assim, a segunda métrica para medir o grau de alisamento nas empresas abertas brasileiras é:

$$
I A 2=\frac{\sigma\left(\text { lucro operacional }_{i t}\right)}{\sigma\left(\text { fluxo de caixa operacional }_{i t}\right)}
$$

\section{METODOLOGIA}

O presente trabalho analisa a relação empírica entre o income smoothing, o custo de capital de terceiros e a estrutura de capital das empresas abertas brasileiras. O modelo na análise foi ajustado daquele proposto por Arellano (2003). Nesta pesquisa, foi utilizado um modelo generalizado de regressão múltipla em dados em painel tipo Nerlove. De forma concreta, será usado um modelo de ajuste parcial que nos permita tratar de relações que contenham valores defasados da variável a testar, que neste estudo é o fenômeno do income smoothing.

$\mathrm{O}$ ponto de partida no desenho do modelo vem marcado pelo objetivo do trabalho: verificar se a gestão da empresa orienta as decisões da estrutura de capital e do custo de capital de terceiros com o propósito de buscar um determinado nível de endividamento, que se estabelece como meta e que se cifra no ratios de endividamento e no custo de capital de terceiros. Em cada período, a gestão espera ajustar a estrutura de capital e o custo de capital de terceiros ao objetivo desejado, mas, por causa das restrições internas e externas que condicionam a gestão financeira da empresa, tais ajustes se produzem apenas parcialmente. Há restrições internas - como o crescimento da empresa, a política de autofinanciamento e a prática do income smoothing - e restrições externas - como a atitude dos fornecedores de capital, as imperfeições do mercado de capitais, as políticas governamentais etc. 
Todos esses fatores em conjunto constituem restrições identificadas que influenciam a política de endividamento e custo de capital examinados.

\subsection{HIPÓTESES}

As hipóteses de pesquisa foram construídas com base no referencial teórico e na disponibilidade de dados. As hipóteses envolvem variáveis de controle que têm sido fatores explicativos da estrutura de capital e do custo de capital de terceiros das empresas: rentabilidade, risco, tamanho, composição dos ativos, crescimento, alavancagem financeira e algumas anomalias de mercado (lucro/preço e Ebitda/preço). Dessa forma, pode-se contrastar empiricamente o alisamento de resultados.

\subsection{DADOS}

O estudo foi feito com base no banco de dados da Economática e da Comissão de Valores Mobiliários (CVM), englobando informações contábeis sobre 2I7 empresas abertas brasileiras, classificadas num primeiro estágio como smoothers e non smoothers, com base no critério de Eckel (I98I), e, num segundo estágio, mensurando o grau quantitativo de alisamento segundo o critério de Leuz, Nanda e Wysocki (2003). O período de estudo correspondeu aos anos de 2003 a 2007. Num primeiro estágio, foram consideradas as observações referentes às empresas que dispunham de todos os dados necessários para o cálculo dos critérios de alisamento e variáveis independentes, ainda que não necessariamente de todos os valores das variáveis independentes utilizadas. Igualmente, também não foram excluídas as observações com valores superiores a três desvios padrão (outliers), conforme sugerido por Tinoco (2007), quando utilizada a metodologia de dados em painel. Dessa forma, o banco de dados final utilizado nos testes econométricos englobou 4.724 observações.

\subsection{VARIÁVEIS}

As variáveis de controle submetidas aos procedimentos econométricos foram definidas considerando o referencial teórico, as pesquisas empíricas já realizadas.

As variáveis dependentes que se referem à estrutura de capital das empresas foram representadas por índices de endividamento calculados a partir dos dados contábeis. Apuraram-se três indicadores de endividamento: uma variável como medida de endividamento de curto prazo (CIRC_AT), uma como medida de endividamento de longo prazo (ELP_AT) e uma como medida de endividamento total (EXIG_PL). Dessa forma, as variáveis dependentes são: 
- CIRC_AT: $Y_{\mathrm{I}}=$ Passivo circulante/ativo total

- ELP_AT: $Y_{2}=$ Exigível de longo prazo/ativo total

- $\quad$ EXIG_PL: $Y_{3}=($ Passivo circulante + exigível de longo prazo $) /$ patrimônio líquido

A variável dependente referente ao custo de capital de terceiros das empresas foi estimada após o efeito dos impostos e tendo presente a porcentagem de cada uma das dívidas.

Para as variáveis independentes que se referem aos fatores considerados pela literatura como potenciais determinantes da estrutura de capital das empresas, foram calculadas métricas para serem utilizadas como proxies. As variáveis utilizadas como representantes de cada um dos fatores foram aquelas que apresentaram os maiores coeficientes de correlação com as variáveis dependentes. Assim, para os fatores rentabilidade, risco, tamanho, composição dos ativos, crescimento e alisamento, as medidas selecionadas são apresentadas no Quadro I.

No tocante ao resultado esperado para a variável smoothing, espera-se que, à medida que a empresa alise menos (índice de alisamento de Leuz alto), diminua a proporção de endividamento de terceiros ou, em outros termos, uma empresa que alise mais (índice de alisamento de Leuz baixo) aumenta a proporção de endividamento de terceiros. Em apertada síntese, espera-se que as empresas que promovem smoothing reduzam a percepção de riscos, passando progressivamente a privilegiar, também de forma progressiva, o financiamento com capital de terceiros.

\section{QUADRO I}

VARIÁVEIS INDEPENDENTES PARA O CONTRASTE DA ESTRUTURA DE CAPITAL

\begin{tabular}{llll}
\hline FATORES & $B$ & PROXIES & SINAL ESPERADO \\
\hline Rentabilidade & $B_{1}$ & Lucro líquido/patrimônio líquido & + \\
\hline Risco & $B_{2}$ & Beta & + \\
\hline Tamanho & $B_{3}$ & Ln vendas & - \\
\hline Permanente & $B_{4}$ & Ativo permanente/ativo total & - \\
\hline Crescimento & $B_{5}$ & Crescimento das vendas (vendas $/$ / vendas ${ }_{t-1}$ ) & \\
\hline Smoothing & $B_{6}$ & $\begin{array}{l}\text { Valor do índice de alisamento de Leuz com uma e } \\
\text { duas defasagens }\end{array}$ & \\
\hline
\end{tabular}

Fonte: Elaborado pelos autores. 
Para as variáveis independentes que se referem aos fatores considerados pela literatura como potenciais determinantes do custo de capital de terceiros das empresas, foram calculadas métricas para serem utilizadas como proxies dos fatores. As variáveis utilizadas como representantes de cada um dos fatores foram aquelas que apresentaram os maiores coeficientes de correlação com as variáveis dependentes. Assim, para os fatores risco, alavancagem, tamanho, anomalias do mercado e alisamento, as medidas selecionadas são apresentadas no Quadro 2.

\section{QUADRO 2}

VARIÁVEIS INDEPENDENTES PARA O CONTRASTE DO CUSTO DE CAPITAL DE TERCEIROS

\begin{tabular}{llll}
\hline FATORES & $B$ & PROXIES & SINAL ESPERADO \\
\hline Risco & $B_{1}$ & Lucro líquido/patrimônio líquido & + \\
\hline $\begin{array}{l}\text { Alavancagem } \\
\text { financeira }\end{array}$ & $B_{2}$ & Beta & + \\
\hline $\begin{array}{l}\text { Tamanho } \\
\text { Anomalias do } \\
\text { mercado }\end{array}$ & $B_{3}$ & Ln vendas & $\begin{array}{l}\text { Relação Ebitda/preço } \\
\text { Ebitda da ação anual/preço de mercado da ação } \\
\text { anual }\end{array}$ \\
\hline $\begin{array}{l}\text { Anomalias do } \\
\text { mercado }\end{array}$ & $B_{5}$ & $\begin{array}{l}\text { Relação preço/lucro } \\
\text { Lucro disponível por ação anual /preço de mercado } \\
\text { da ação anual }\end{array}$ & + \\
\hline $\begin{array}{l}\text { Smoothing } \\
\text { Valor do índice de alisamento de Leuz com uma e } \\
\text { duas defasagens }\end{array}$ & +
\end{tabular}

Fonte: Elaborado pelos autores.

\subsection{PROCEDIMENTOS ESTATÍSTICOS}

Quando se estuda o comportamento de um conjunto de dados em conglomerados ao longo de determinado período, devem-se considerar as diferenças entre as empresas e as especificidades de cada período que compõem a amostra. A estimação de um modelo que utilize a estrutura de dados em painel possibilita o desenvolvimento da análise levando em consideração tanto as características individuais ao longo do tempo quanto os fatores atribuídos a determinados períodos e que afetam igualmente as empresas naquele momento. Segundo Marques (2000), uma das vantagens da estimação por dados em painel é a revelação da heterogeneidade 
dos indivíduos amostrais. Outras vantagens listadas pelo autor são: maior quantidade de informação, maior variabilidade dos dados, menor colinearidade entre as variáveis, maior número de graus de liberdade e maior eficiência na estimação.

Anteriormente, Beaver et al. (I989) já haviam se manifestado com relação às vantagens do panel data com efeitos fixos que permite I. combinar a análise da série-temporal com a análise de corte transversal em um mesmo teste de significância; 2. estimar resíduos com baixa correlação, gerando uma melhora da estimação e de erros padrões não enviesados; e 3. apresentar coeficientes de inclinação da reta menos sujeitos ao viés decorrente da omissão de variáveis.

Assim, o presente estudo utiliza a metodologia de dados em painel, onde $i$ expressa a quantidade de empresas que compõem a amostra, e $t$, o número de anos observados. Quando o número de observações for igual ao número de unidades transversais, o painel de dados será considerado completo (balanceado); caso contrário, será considerado incompleto (não balanceado). A presente pesquisa trabalha com dados em painel não balanceados, uma vez que são analisadas 2I7 empresas durante um período de 5 anos. Conforme foi citado anteriormente, a estrutura de dados em painel possibilita o desenvolvimento da análise levando em consideração as características individuais ao longo do tempo e os efeitos de fatores relacionados ao período sobre as empresas.

Para controlar os efeitos não observáveis de forma individual, supõe-se que: $\varepsilon_{i t}=\alpha_{1}+v_{i t}$ : onde $\alpha_{1}$ absorve toda a heterogeneidade transversal e $v_{i t}$ representa o termo de perturbação estocástico. O modelo proposto utiliza efeitos fixos, tanto individuais quanto temporais, para capturar as especificidades de cada empresa que compõem a amostra e também aquelas relativas ao período, que tem influência sobre todas as empresas analisadas.

Cabe ressaltar que, segundo Hsiao (2004), Arellano (2003) e Wooldridge (2003), a análise de dados em painel permite que o termo de perturbação estocástico $v_{i t}$ seja heterocedástico, autocorrelacionado e que possa existir correlação cruzada entre os grupos, em razão das características da base amostral, cujos períodos analisados são relativamente maiores que as unidades transversais observadas.

O estudo envolveu um conjunto de quatro regressões, sendo três as métricas de endividamento e uma referente ao custo de capital de terceiros. Os dados foram tratados por meio do software Eviews, versão 5.I, e o modelo matemático utilizado foi o seguinte:

$$
\begin{aligned}
& Y_{1}=\alpha+\beta_{1} \operatorname{Re} n t_{i}+\beta_{2} \text { Risc }_{i}+\beta_{3} \text { Tam }_{i}+\beta_{4} \text { Perm }_{i}+\beta_{5} \text { Cresc }_{i}+\beta_{6} \text { Smoothing }_{t-1}+\beta_{7} \text { Smoothing }_{t-2}+\varepsilon_{i} \\
& Y_{2}=\alpha+\beta_{1} \operatorname{Rent}_{i}+\beta_{2} \text { Risc }_{i}+\beta_{3} \text { Tam }_{i}+\beta_{4} \text { Perm }_{i}+\beta_{5} \text { Cresc }_{i}+\beta_{6} \text { Smoothing }_{t-1}+\beta_{7} \text { Smoothing }_{t-2}+\varepsilon_{i} \\
& Y_{3}=\alpha+\beta_{1} \operatorname{Re} n t_{i}+\beta_{2} \text { Risc }_{i}+\beta_{3} \text { Tam }_{i}+\beta_{4} \text { Perm }_{i}+\beta_{5} \text { Cresc }_{i}+\beta_{6} \text { Smoothing }_{t-1}+\beta_{7} \text { Smoothing }_{t-2}+\varepsilon_{i} \\
& Y_{4}=\alpha+\beta_{1} \text { Beta }_{i}+\beta_{2} \text { Alavanc }_{i}+\beta_{3} \text { Tam }_{i}+\beta_{4} \text { Lucro }_{\text {preş }}+\beta_{5} \text { EBITDA A }_{\text {preso }}+\beta_{6} \text { Smoothing }_{t-1}+\beta_{7} \text { Smoothing }_{t-2}+\varepsilon_{i}
\end{aligned}
$$




\subsection{TESTE DE ROBUSTEZ}

Com a finalidade de averiguar a robustez dos resultados, foram realizados testes para verificar a existência de raízes unitárias nas séries e de autocorrelação nos termos residuais. A estacionariedade é uma propriedade que deve ser observada quando se analisa uma série temporal. Segundo Stock e Watson (2000, p. 7I9), o processo estacionário estocástico se caracteriza pelo fato de sua média e variância permanecerem constantes ao longo do tempo e pelo valor da covariância entre dois períodos ser dependente da defasagem entre os dois períodos considerados, e não do período em que é calculada. A violação dessa propriedade, no entendimento de Wooldridge (2003, p. 367), pode influenciar o comportamento da série temporal, resultando em regressões espúrias.

Para testar a estacionariedade do modelo, são analisados os resultados dos testes de raízes unitárias. A análise levou em consideração os resultados do teste de Dickey-Fuller aumentado (ADF) e do teste de Phillips-Perron (PP). Uma das hipóteses do modelo clássico de regressão linear é que não há autocorrelação ou correlação serial nos termos residuais. Na presença de autocorrelação, os estimadores de mínimos quadrados ordinários (OLS) permanecem não lineares, não enviesados e consistentes, entretanto deixam de ser eficientes. Conforme Stock e Watson (2003, p. 4II), nesses casos, os estimadores deixam de ser os de menor variância, ou seja, os estimadores deixam de ser considerados como melhor estimador linear não enviesado, e, consequentemente, os resultados gerados pela regressão deixam de ser confiáveis. Para verificar a presença de autocorrelação, foi utilizado o teste de Durbin-Watson, cujos resultados são apresentados com os resultados da análise da regressão.

\section{ANÁLISE DOS RESULTADOS}

Nesta seção, são apresentados e analisados os resultados das regressões. Os coeficientes estimados pelos modelos foram considerados robustos, fundamentados na análise de dois testes estatísticos. Primeiro, pela consistência dos modelos, considerando-se os critérios de informação de Akaike e Schwartz. Segundo, pela significância estatística de as variáveis se manterem, quase sempre, acima de $95 \%$, em valores absolutos. 


\section{ANÁLISE DOS RESULTADOS ESTIMADOS DOS MODELOS REFERENTES À ESTRUTURA DE CAPITAL}

Para testar as hipóteses de pesquisa, foi necessário, em princípio, estimar o componente do fator smoothing defasado para um e dois períodos, a partir dos modelos estimados. As variáveis explanatórias utilizadas no modelo identificam os fatores indicados pela teoria como explicativos da estrutura de capital e do custo de capital de terceiros. Os resultados dos modelos referentes à estrutura de capital são apresentados nas tabelas I, 2 e 3.

Esses resultados evidenciam que as variáveis que mais contribuem para explicar os indicadores de endividamento das empresas são risco (Risco), composição dos ativos (Perm) e alisamento (Smoothing), cujos coeficientes são estatisticamente significativos nas três regressões. As variáveis tamanho (TAM) e crescimento (Cresc) se mostraram determinantes dos endividamentos de longo prazo (ELP_AT) e total (EXIG_PL), não sendo significativos para o endividamento de curto prazo. Não se mostraram significativas para nenhum dos indicadores de endividamento considerados a variável rentabilidade (Rent).

\section{TABELA I}

\section{RESUMO DOS TESTES ESTATÍSTICOS}

\begin{tabular}{llcc} 
MODELO $1 Y_{1}=\alpha+\beta_{1}$ Rent $_{i}+\beta_{2}$ Risc $_{i}+\beta_{3}$ Tam $_{i}+\beta_{4}$ Perm $_{i}+\beta_{5}$ Cresc $_{i}+\beta_{6}$ Smoothing $_{t-1}+\beta_{7}$ Smoothing $_{t-2}+\varepsilon_{i}$ \\
\hline Variável dependente: & CIRC_AT & & \\
Método & Dados em painel com efeitos fixos & \\
Período considerado: & $2003-2007$ & & \\
Empresas analisadas: & 248 & & \\
Total de observações: & 2547 & & \\
\hline VARIÁVEIS SIGNIFICATIVAS & COEFICIENTE & ESTATISTICA t & P-VALOR \\
\hline RISCO & 0,0045 & 4,214 & 0,001 \\
PERMANENTE & $-0,568$ & $-21,365$ & 0,001 \\
SMOOTHING t-1 & $-0,0038$ & $-5,214$ & 0,0324 \\
\hline R2 & 0,389 & & \\
R 2 Ajustado & 0,351 & & \\
F-Estatístico & 14,562 & & \\
Critério de Informação de Akaike & $-3145,321$ & & \\
Critério de Informação de Scwartz & $-3214,208$ & & \\
P-valor (F-Estatístico) & 0,0000 & & \\
\hline
\end{tabular}


Em termos gerais, a regressão envolvendo a variável independente CIRC_AT foi a que apresentou o maior coeficiente de determinação ajustado $\left(R^{2}\right.$ ajustado $=$ 0,35I) e critérios de informação válidos para as variáveis explanatórias, indicando que os fatores estudados explicam mais a variação do endividamento de curto prazo do que as variações dos endividamentos de longo prazo.

De forma específica, os resultados das regressões mostram que os sinais dos coeficientes do fator smoothing é negativo para os três modelos, confirmando a expectativa fundamentada de que a prática de alisamento elevaria a capacidade de captar volume de dívida pelas empresas. As estatísticas $t$, com $p$-valor significativos, comprovam que a variável smoothing explica o comportamento da estrutura de capital de curto e longo prazos, assim como o endividamento total. O fator smoothing, analisado por meio de suas defasagens estaria indicando que a prática de alisamento sinaliza estabilidade, promovendo o aumento do endividamento por parte da empresa através dos anos, já que essa prática está sendo significativa até com dois períodos de retardos.

\section{TABELA 2}

\section{RESUMO DOS TESTES ESTATISTICOS}

\begin{tabular}{|c|c|c|c|}
\hline \multicolumn{4}{|c|}{ MODELO $2 Y_{2}=\alpha+\beta_{1}$ Rent $_{i}+\beta_{2}$ Risc $_{i}+\beta_{3}$ Tam $_{i}+\beta_{4}$ Perm $_{i}+\beta_{5}$ Cresc $_{i}+\beta_{6}$ Smoothing $_{t-1}+\beta_{7}$ Smoothing $_{t-2}+$} \\
\hline Variável dependente: & ELP_AT & & \\
\hline Método & Dados em paine & $m$ efeitos fixos & \\
\hline Período considerado: & 2003-2007 & & \\
\hline Empresas analisadas: & 284 & & \\
\hline Total de observações: & 2988 & & \\
\hline VARIÁVEIS SIGNIFICATIVAS & COEFICIENTE & ESTATÍSTICA t & $P$-VALOR \\
\hline RISCO & 0,0215 & 5,311 & 0,00000 \\
\hline TAMANHO & 0,981 & 2,694 & 0,00000 \\
\hline PERMANENTE & 0,567 & 8,214 & 0,00000 \\
\hline CRESCIMENTO & 0,0004 & 6,214 & 0,001 \\
\hline SMOOTHING t-1 & $-0,0021$ & $-3,257$ & 0,0024 \\
\hline $\mathrm{R}^{2}$ & 0,350 & & \\
\hline $\mathrm{R}^{2}$ Ajustado & 0,328 & & \\
\hline F-Estatístico & 17,065 & & \\
\hline Critério de Informação de Akaike & $-3441,271$ & & \\
\hline Critério de Informação de Scwartz & $-4011,102$ & & \\
\hline P-valor (F-Estatístico) & 0,0000 & & \\
\hline
\end{tabular}


TABELA 3

RESUMO DOS TESTES ESTATÍSTICOS

\begin{tabular}{lccc}
\hline MODELO $3 Y_{3}=\alpha+\beta_{1}$ Rent $_{i}+\beta_{2}$ Risc $_{i}+\beta_{3}$ Tam $_{i}+\beta_{4}$ Perm $_{i}+\beta_{5}$ CresC $_{i}+\beta_{6}$ Alisamento $_{t-1}+\beta_{7}$ Alisamento $_{t-2}+\varepsilon_{i}$ \\
\hline Variável dependente: & EXIG_PL & & \\
Método & Dados em painel com efeitos fixos & \\
Período considerado: & $2003-2007$ & & \\
Empresas analisadas: & 274 & & \\
Total de observações: & 2751 & & \\
\hline VARIÁVEIS SIGNIFICATIVAS & COEFICIENTE & ESTATísTICA t & P-VALOR \\
\hline RISCO & 0,03015 & 6,324 & 0,0010 \\
TAMANHO & 0,841 & 3,514 & 0,0000 \\
PERMANENTE & $-0,652$ & $-2,987$ & 0,0000 \\
CRESCIMENTO & 0,0014 & 3,012 & 0,0010 \\
SMOOTHING t-1 & $-0,0034$ & $-2,957$ & 0,0240 \\
SMOOTHING t-2 & $-0,00029$ & $-3,247$ & 0,0000 \\
\hline R2 & 0,331 & & \\
R² Ajustado & 0,284 & & \\
F-Estatístico & 19,254 & & \\
Critério de Informação de Akaike & $-3879,231$ & & \\
Critério de Informação de Scwartz & $-3587,257$ & & \\
P-valor (F-Estatístico) & 0,0001 & & \\
\hline
\end{tabular}

Fonte: Elaborado pelos autores.

\subsection{ANÁLISE DO RESULTADO DOS MODELOS ORIENTADOS AO CUSTO DE CAPITAL DE TERCEIROS}

Os resultados referentes à relação custo de capital de terceiros e o alisamento de resultados evidenciam que o fator smoothing pode explicar o indicador do custo de capital de terceiros das empresas. Foram testados diferentes modelos de regressão utilizando como variável independente o indicador Ke. Nesse caso, buscou-se escolher uma variável de cada grupo para as regressões. A regressão apresentada na Tabela 4 foi a que apresentou os melhores resultados em termos de explicação da variável dependente, bem como de significância das variáveis explicativas. Os determinantes escolhidos explicam 37,8\% do custo de capital de terceiros, sendo todos significativos a $5 \%$ pelo menos.

Quanto ao fator smoothing, espera-se, a priori, uma relação positiva com o custo de capital de terceiros. O motivo é que as empresas que têm um perfil de lucros menos variável podem ser vistas como tendo menos risco, reduzindo o 
custo de capital. Dessa forma, como existe uma relação positiva e significativa a I\% entre o fator smoothing com uma e duas defasagens e custo de capital de terceiros, seria um indicativo de que a prática de alisamento por parte das empresas brasileiras teria o impacto de diminuir o custo de capital de terceiros.

\section{TABELA 4}

\section{RESUMO DOS TESTES ESTATÍSTICOS}

\begin{tabular}{|c|c|c|c|}
\hline \multicolumn{4}{|c|}{$\beta_{7}$ Smoothing $_{t-2}+\varepsilon_{i}$} \\
\hline Variável dependente: & $\mathrm{Ke}$ & & \\
\hline Método & Dados em paine & $n$ efeitos fixos & \\
\hline Período considerado: & 2003-2007 & & \\
\hline Empresas analisadas: & 304 & & \\
\hline Total de observações: & 3694 & & \\
\hline VARIÁVEIS SIGNIFICATIVAS & COEFICIENTE & ESTATÍSTICA t & $P$-VALOR \\
\hline RISCO & 0,00025 & 3,598 & 0,0010 \\
\hline ALAVANCAGEM FINANCEIRA & 0,00147 & 4,367 & 0,0000 \\
\hline TAMANHO & $-0,00267$ & $-6,587$ & 0,0010 \\
\hline EBITDA/PREÇO & 0,0245 & 4,687 & 0,0010 \\
\hline SMOOTHING t-1 & 0,0068 & 3,014 & 0,0310 \\
\hline SMOOTHING t-2 & 0,0249 & 4,698 & 0,0000 \\
\hline $\mathrm{R}^{2}$ & 0,394 & & \\
\hline $\mathrm{R}^{2}$ Ajustado & 0,378 & & \\
\hline F-Estatístico & 20,698 & & \\
\hline Critério de Informação de Akaike & $-4254,368$ & & \\
\hline Critério de Informação de Scwartz & $-4587,957$ & & \\
\hline P-valor (F-Estatístico) & 0,0001 & & \\
\hline
\end{tabular}

Fonte: Elaborado pelos autores.

\subsection{RESULTADOS DOS TESTES DE ROBUSTEZ APLICADOS AOS MODELOS}

A seguir, são apresentados os resultados dos testes de robustez aplicados aos modelos de regressão utilizados. Para verificar a estacionariedade das séries temporais, foram realizados os testes de raízes unitárias. A Tabela 5 apresenta os resultados dos testes de Dickey-Fuller aumentado (ADF) e de Phillips-Perron (PP). A hipótese nula dos testes ADF e PP é que as séries analisadas possuem raízes unitárias. As estatísticas calculadas pelos testes ADF e PP para os quatro 
modelos analisados, ambos com $p$-valor $(0,0 \circ 0)$, rejeitam a hipótese nula. Esses resultados comprovam, estatisticamente, que as séries temporais analisadas são estacionárias, sinalizando a robustez dos modelos utilizados para testar o fator smoothing na estrutura de capital e no custo de capital de terceiros.

O segundo teste de robustez aplicado ao modelo visa verificar se os resíduos do modelo possuem autocorrelação, o que, se for confirmado, constitui violação a uma das hipóteses da metodologia OLS. Foi utilizado o teste de Durbin-Watson para essa finalidade. Portanto, a hipótese nula a ser testada é a de que os resíduos da regressão são autocorrelacionados. Os resultados da estatística $d$ para os quatro modelos analisados, apresentados na Tabela 6 , rejeitam a hipótese de autocorrelação dos resíduos. Pela análise, conclui-se que os estimadores podem ser considerados como melhor estimador linear não enviesado (Melne), resultados que confirmam a robustez dos modelos utilizados.

\section{TABELA 5}

TESTE DE RAÍZ UNITÁRIA DOS MODELOS DE REGRESSÃO

\begin{tabular}{lcc}
\hline MODELO 1 & & \\
\hline MÉTODO & ESTATÍSTICA & P-VALOR \\
\hline ADF - Fisher Qui-quadrado & $5.487,37$ & 0,000 \\
PP - Fisher Qui-quadrado & $7.214,28$ & 0,000 \\
\hline MODELO 2 & & \\
\hline MÉTODO & ESTATÍSTICA & P-VALOR \\
\hline ADF - Fisher Qui-quadrado & $4.982,25$ & 0,000 \\
PP - Fisher Qui-quadrado & $6.489,22$ & 0,000 \\
\hline MODELO 3 & & P-VALOR \\
\hline MÉTODO & ESTATÍSTICA & 0,000 \\
\hline ADF - Fisher Qui-quadrado & $5.045,67$ & 0,000 \\
PP - Fisher Qui-quadrado & $5.002,67$ & \\
\hline MODELO 4 & & P-VALOR \\
\hline MÉTODO & & 0,000 \\
\hline ADF - Fisher Qui-quadrado & ESTATÍSTICA & 0,000 \\
PP - Fisher Qui-quadrado & $7.598,36$ & \\
\hline
\end{tabular}




\section{TABELA 6}

TESTE DE DURBIN-WATSON DOS MODELOS DE REGRESSÃO

\begin{tabular}{lc}
\hline MODELO 1 & \\
\hline TESTE & ESTATISTICA \\
\hline Durbin-Watson & 2,368 \\
\hline MODELO 2 & ESTATÍSTICA \\
\hline TESTE & 2,104 \\
\hline Durbin-Watson & \\
\hline MODELO 3 & ESTATISTICA \\
\hline TESTE & 2,387 \\
\hline Durbin-Watson & \\
\hline MODELO 4 & ESTATÍSTICA \\
\hline TESTE & 2,0041 \\
\hline Durbin-Watson
\end{tabular}

Fonte: Elaborado pelos autores.

\section{CONSIDERAÇÕES FINAIS}

A presente pesquisa analisou as características da estrutura de capital e do custo de capital de terceiros das empresas abertas brasileiras, investigando as relações existentes entre o nível de endividamento, seu custo e sua relação com o income smothing, mantendo os fatores apontados pela teoria financeira como fatores de controle. Os resultados dos testes aplicados indicaram que o income smoothing é determinante para a estrutura de capital. Quanto ao custo de capital de terceiros das empresas analisadas, o fator smoothing se mostra significativo com uma e duas defasagens, o que estaria indicando que o gestor está olhando o presente e o passado da estrutura de capital e do custo de capital de terceiros.

Entre as principais características diferenciais deste estudo, destaque-se a utilização de um modelo de regressão em dados em painel que permitiu controlar a heterogeneidade estrutural das empresas ao longo das séries de anos, bem como trabalhar com uma amostra maior de empresas. Os modelos de regressão apontam que os fatores analisados neste trabalho explicam apenas parte das 
variações no endividamento das empresas e do custo de capital de terceiros, o que sugere que outras variáveis também influenciam a forma como as empresas captam dívida no Brasil. Entre essas variáveis, podem ser destacadas as de fatores comportamentais específicos dos gestores, além de outros aspectos relacionados às características do ambiente econômico e institucional brasileiro e não controladas pelo modelo de regressão em dados em painel.

As conclusões deste trabalho são importantes para compreensão dos fatores que explicam a definição do custo de capital e, consequentemente, da estrutura de capital. Essas variáveis são cruciais para avaliação de qualquer empresa.

\section{REFERÊNCIAS}

ALBRECHT, W. D.; RICHARDSON, F. M. Income smoothing by economy sector. Journal of Business Finance and Accounting, v. 7, n. 5, p. 713-730, Winter I990.

ARELLANO M. Panel data econometrics. Oxford: Oxford University Press, 2003.

ASHARI, N. et al. Factors affecting income smoothing among listed companies in Singapore. Accounting and Business Research, v. 24, n. 96, p. 29I-301, I994.

BAO, B. Income smoothing, earnings quality and firm valuation. Journal of Business Finance and Accounting, v. 3I, 2004 .

BARNEA, A.; RONEN, J.; SADAN, S. Classificatory smoothing of income with extraordinary items. The Accounting Review, v. 5I, n. I, p. IIo-I22, Jan. I976.

BARTH, M. E.; LANDSMAN W. R.; WAHLEN, J. M. Fair value accounting: effects on banks'earnings volatility, regulatory capital and value of contractual cash flows. Journal of Banking and Finance, v. I9, p. 577-605, I995.

BEATTIE, V. et al. Extraordinary items and income smoothing: a positive accounting approach. Journal of Business Finance and Accounting, v. 2I, n. 6, p. 79I-8II, I994.

BEAVER, W. et al. Financial reporting and the structure of bank share prices. Journal of Accounting Research, v. 207, n. 2, p. 157-I78, I989.

BEIDLEMAN, C. R. Income smoothing: the role of management. The Accounting Review, v. 48, n. 4, p. 653-667, I973.

BIN, K; WAN B.; KAMIL, K. Market perception of income smoothing practices: Malaysian evidence. Journal of Economics and Finance, v. 26, n. 2, p. I32-I46, Spring 2000.

BOOTH, G. G.; KALLUNKI, J. P.; MARTIKAINEN, T. Post-announcement drift and income smoothing: finnish evidence. Journal of Business Finance and Accounting, v. 23, n. 8, p. II97-I2II, Oct. I996.

CHALAYER, S. Identification et motivations des pratiques de lissage dês résultats comptables des entreprises françaises cotées en Bourse. I992. Thèse (Doctorat)-Université de Saint-Etienne.

COLLINS, D. W.; KOTHARI, S. P. An analisis of intertemporal and cross-sectional determinants of earnings response coefficients. Journal of Accounting and Economics, v. II, n. 2-3, p. I43-I82, I989. COPELAND, R. M. Income smoothing. Empirical Research in Accounting, p. IOI-II6, I968. Selected Studies 6 (Supplement). 
EASTON, P.; ZMIJEWSKI, M. Cross-sectional variance in the stock market response to accounting earnings measurements. Journal of Accounting and Economics, v. II, p. II7- I42, I989.

ECKEL, N. The income smoothing hypothesis revisited. Abacus, v. I7, n. I, p. 28-40, I98I.

FRANCIS, J. R., MAYDEW, E. L.; SPARKS, H. C. The role of big 6 Auditors in the credible reporting of accruals. Auditing: a Journal of Practice and Theory, v. I8, p. I7-34, 2004.

GIBBINS, M.; RICHARDSON, A.; WATERHOUSE, J. The management of corporate financial disclosure: opportunism, ritualism, policies, and process. Journal of Accounting Research, v. 28, p. I2I-I43, Spring I990.

GORDON, M. J. Postulates, principles and research in accounting. The Accounting Review, v. 39, p. 25I-263, I964.

GRANT, M.; PARBONETTI, E. CEO Risk-Related Incentives and Income Disponível em: <SSRN. com/abstract=975266>. Acesso em: I out. 2007.

HSIAO, C. Analysis of panel data. Oxford: Oxford University Press, 2004.

IMHOFF, E. A. Income smoothing: an analysis of critical issues. Quarterly Review of Economics and Business, v. 2I, n. 3, p. 23-42, I98I.

IÑIGUEZ, R.; POVEDA, F. Long-run abnormal returns and income smoothing in the spanish stock market. European Accounting Review, v. I3, n. I, p. I05-130, 2004.

LEUZ, C.; NANDA, D.; WYSOCKI, P. Earnings management and investors protection: an international comparison. Journal of Financial Economics 69, v. 3, p. 505-527, Sept. 2003.

LOPES, A . B.; TUKAMOTO, Y. S.; GALDI, F. C. Earnings management and cross listing in Brazil. In: CONGRESSO ANPCONT, I., 2007, Brasil.

MARQUES, L. D. Modelos dinâmicos com dados em painel: revisão de literatura. Porto: Centro de Estudos Macroeconômicos e Previsão da Faculdade de Economia do Porto, out. 2000. Disponível em: <http://www.fep.up.pt/investigacao/workingpapers>. Acesso em: 20 dez. 2008.

MICHELSON, S. E., JORDAN-WAGNER, J.; WOOTTON, C. W. A market based analysis of income smoothing. Journal of Business Finance and Accounting, v. 22, n. 8, p. II79-II93, Dec. I995.

The relationship between the smoothing of reported income and risk-adjusted returns. Journal of Economics and Finance, v. 24, n. 2, p. I4I-I59, Summer I999.

RONEN, J.; TZUR, J.; YAARI, V. Legal insider trading, CEO's incentive, and quality of earnings. Corporate Governance and Control, v. 4, p. 210-2I9, Spring, 2007.

STOCK, J. H.; WATSON, M. W. Econometria. São Paulo: Pearson Education Brasil, 2000.

STOLOWY, H.; BRETON, G. A. Review of reserach on accounts manipulation. In: ANNUAL CONGRESS OF THE EUROPEAN ACCOUNTING ASSOCIATION, 23., Munich, March 2000, EAA (European Accounting Association).

TAN, E.; JAMAL, A. Effects of accounting discretion on abilitu of managers to smooth earnings. Journal of Accounting and Public Policy, v. 25, n.5, p. 554-573, Sept./Oct. 2006.

TINOCO. M. Análise estatística. Lisboa: Edições Sílabo, 2007.

TRUEMAN, B.; TITMAN, S. An explanation for accounting income smoothing. Journal of Accounting Research, v. 26, p. 127-I39, I988. Supplement.

TUCKER, E.; ZAROWIN, J. Does income smoothing improve earnings informativeless. The Accounting Review, v. 86, n. I, 2006.

WOOLDRIDGE, J. Econometric analysis cross-section and panel data. Oxford: Oxford University Press, 2003. 